\title{
Clinical evaluation of right recurrent laryngeal nerve nodes in thoracic esophageal squamous cell carcinoma
}

\author{
Zhen-Xuan Li ${ }^{1 \#}$, Xiao-Dong $\mathrm{Li}^{2 \#}$, Xian-Ben Liu ${ }^{1}$, Wen-Qun Xing ${ }^{1}$, Hai-Bo Sun ${ }^{1}$, Zong-Fei Wang ${ }^{1}$, \\ Rui-Xiang Zhang', Yin Li,
}

${ }^{1}$ Department of Thoracic Surgery, The Affiliated Cancer Hospital of Zhengzhou University, Zhengzhou, China; ${ }^{2}$ Department of Thoracic Surgery, Sun Yat-sen University Cancer Center, State Key Laboratory of Oncology in South China, Guangzhou, China; ${ }^{3}$ Department of Thoracic Surgery, National Cancer Center/Cancer Hospital, Chinese Academy of Medical Sciences and Peking Union Medical College, Beijing, China Contributions: (I) Conception and design: Y Li, ZX Li; (II) Administrative support: None; (III) Provision of study materials or patients: None; (IV) Collection and assembly of data: XD Li, ZX Li; (V) Data analysis and interpretation: ZX Li; (VI) Manuscript writing: All authors; (VII) Final approval of manuscript: All authors.

"These authors contributed equally to this work.

Correspondence to: Yin Li. MD, PhD. Department of Thoracic Surgery, The Affiliated Cancer Hospital of Zhengzhou University, 127 Dongming Rd, Zhengzhou 450008, China. Email: liyin825@sina.com.

Background: The accuracy of clinical $\mathrm{N}$ staging of esophageal squamous cell carcinoma is suboptimal. As an important station of lymph node metastasis, station C201 (right recurrent laryngeal nerve nodes) has rarely been evaluated alone. We aimed to explore an effective way to evaluate the right recurrent laryngeal nerve nodes in thoracic esophageal squamous cell carcinoma.

Methods: We retrospectively analyzed 628 thoracic esophageal squamous cell carcinoma patients who underwent radical resection without neoadjuvant therapy from two Chinese cancer centers. The diameter of the short axis of the largest right recurrent laryngeal nerve node (DC201) was measured on contrastenhanced multi-slice computed tomography (MSCT). Right recurrent laryngeal nerve nodes were examined by postoperative pathologic results. The receiver operating characteristic (ROC) curve was generated to assess the diagnostic capabilities of DC201 to determine the right recurrent laryngeal nerve nodes status.

Results: ROC curve analysis revealed that the optimal cut-off point of DC201 was $6 \mathrm{~mm}$, with an area under curve (AUC), sensitivity, specificity, and Youden index of 0.896, 71.9\%, 88.8\%, and 0.607 respectively. When the cut-off point of DC201 was set to $10 \mathrm{~mm}$, sensitivity, specificity and the Youden index were $14.1 \%$, 99.6\% and 0.137 respectively. Among 128 patients with right recurrent laryngeal nerve node metastasis, 71 and 108 patients had the largest right recurrent laryngeal nerve node located above the suprasternal notch level and in the tracheoesophageal groove respectively.

Conclusions: When DC201 $\geq 6.0 \mathrm{~mm}$ instead of DC201 $\geq 10 \mathrm{~mm}$ was used to dictate the right recurrent laryngeal nerve nodes metastasis, contrast-enhanced MSCT could evaluate the status of right recurrent laryngeal nerve nodes with high sensitivity and specificity. The largest right recurrent laryngeal nerve nodes were mainly located in the tracheoesophageal groove and/or above the suprasternal notch.

Keywords: Esophageal squamous cell carcinoma; computed tomography (CT); diagnosis; right recurrent laryngeal nerve nodes; cancer staging

Submitted Feb 06, 2020. Accepted for publication Jun 02, 2020.

doi: $10.21037 /$ jtd-20-774

View this article at: http://dx.doi.org/10.21037/jtd-20-774 


\section{Introduction}

Clinical and pathologic categories of lymph node status for thoracic esophageal squamous cell carcinoma are not considered to denote prognostic implications by the Worldwide Esophageal Cancer Collaboration for the AJCC/UICC cancer staging manuals $8^{\text {th }}$ edition (1-3). For cT1 and cT2 squamous cell carcinoma, no disparities of risk-adjusted survival were noted between $\mathrm{cN} 0$ and $\mathrm{cN} 1$, raising the possibility of low accuracy of clinical $\mathrm{N}$ staging (2). To our knowledge, clinical criteria for lymph node metastasis have not been established rigorously in peer-reviewed literature. In clinical practice, locoregional nodes are generally suspicious for tumor involvement when being round in shape and/or $>10 \mathrm{~mm}$ in the short axis. However, the sizes of normal lymph nodes vary significantly in different stations (4-6), suggesting that adopting flexible criteria for lymph nodes in different stations may improve the accuracy of clinical $\mathrm{N}$ staging.

In the lymph node maps for esophageal cancer, right recurrent laryngeal nerve nodes (station C201) are located along the right recurrent laryngeal nerve that are superiorly and inferiorly bounded by the cephalic border of the right subclavian artery, and the caudal border of the right recurrent laryngeal nerve curving upward respectively (7), which is equivalent to right upper paratracheal nodes (station 2R) in AJCC/UICC classification (8th). Right upper paratracheal nodes are located between the intersection of the caudal margin of the brachiocephalic artery with the trachea and the apex of the lung (8), station C201 has been documented to be the most frequent site of lymph node metastases of thoracic esophageal squamous cell carcinoma (9-11). Moreover, recurrent laryngeal nerve injury that is related to station C201 dissection is a common surgical complication that is associated with aspiration pneumonia (12-15). Thus right recurrent laryngeal nerve nodes in thoracic esophageal squamous cell carcinoma are the focus of thoracic surgeons' attention. The purpose of our study is to explore whether adopting a different criterion improve the clinical diagnostic efficacy of right recurrent laryngeal nerve nodes in thoracic esophageal squamous cell carcinoma. We present the following article in accordance with the STROBE reporting checklist (available at http:// dx.doi.org/10.21037/jtd-20-774).

\section{Methods}

The trial was conducted in accordance with the Declaration of Helsinki (as revised in 2013) and the Harmonized Tripartite Guideline for Good Clinical Practice from the International Conference on Harmonization. This study was reviewed and approved by the Sun Yat-sen University Cancer Center (SYSUCC) and the Affiliated Cancer Hospital of Zhengzhou University (ACHZZU). Informed consents were waived due to its retrospective nature. The electronic medical records of all patients who underwent esophagectomy with extended mediastinal lymph node dissection for thoracic esophageal squamous cell carcinoma at SYSUCC between October 2009 and September 2012, and at ACHZZU between January 2013 and December 2016 were reviewed. Patients who received neoadjuvant therapy were excluded, as were those who had other concomitant cancers and those who did not undergo a resection with curative intent. Patients with incomplete clinical or imaging data were also excluded. Data were collected from 2 different institutions at different time periods due to the work transfer of the author. Furthermore, in order to increase study reliability, we only selected patients whose surgeons performed $>100$ such operations per year. Data from a total of 628 patients were included into the final analysis, including 236 and 392 from SYSUCC and ACHZZU respectively. There was no difference between the two centers in the extent of dissection of right recurrent laryngeal nerve nodes. Surgeons used to open the superior mediastinal pleura along the posterior edge of the trachea, dissociate the posterior wall of the esophagus to the top of the pleura, separate the right recurrent laryngeal nerve along the right subclavian artery, and excise the right recurrent laryngeal nerve nodes together with the adipose tissue. The removed right recurrent laryngeal nerve nodes were placed in a separate container for pathological examination.

Five mm thickness contrast-enhanced multi-slice computed tomography (MSCT) of the chest and abdomen were retrieved from the Picture Archiving and Communication Systems and reviewed (In order to unify standards, we use $5 \mathrm{~mm}$ sliders for assessment). The radiographs were reviewed by a radiologist and a surgeon, and when the results differed, they were discussed and recorded after a consensus was reached. The largest right recurrent laryngeal nerve node in each patient was identified, followed by measurement of its short axis diameter and recoding its location. We defined $\angle \alpha$ in Figure 1, the line between the center of trachea and the center of esophagus is regarded as one side, and the horizontal line is regarded as the other side. Right recurrent laryngeal nerve nodes located in the tracheoesophageal groove were represent by A, Right recurrent laryngeal nerve nodes located 


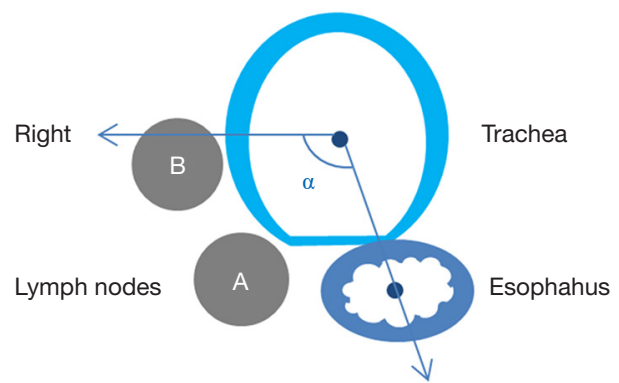

Figure 1 Distribution of metastatic right recurrent laryngeal nerve nodes on the transverse plane. A, Right recurrent laryngeal nerve nodes located in the tracheoesophageal groove; B, Right recurrent laryngeal nerve nodes located on the right side of the trachea away from the esophagus; $\angle \alpha$, The line between the center of trachea and the center of esophagus is regarded as one side, and the horizontal line is regarded as the other side. on the right side of the trachea away from the esophagus were represent by B. Right recurrent laryngeal nerve nodes were divided into the cervical, junctional and thoracic right recurrent laryngeal nerve nodes respectively by the suprasternal notch (Figure 2). Postoperative pathological examinations were used as the gold standard to ascertain the right recurrent laryngeal nerve nodes status. The distances between the lymph nodes and the incisors were retrieved from patients at SYSUCC who routinely underwent endoscopic ultrasonography (EUS). We set the lower boundary of right recurrent laryngeal nerve nodes as $23 \mathrm{~cm}$ from the incisors.

Numerical data were expressed as mean \pm standard deviation or median for randomly and non-randomly distributed data respectively. Categorical variables were presented as frequencies or ratios, as appropriate. The chi-square or Fisher exact tests were applied to analyze
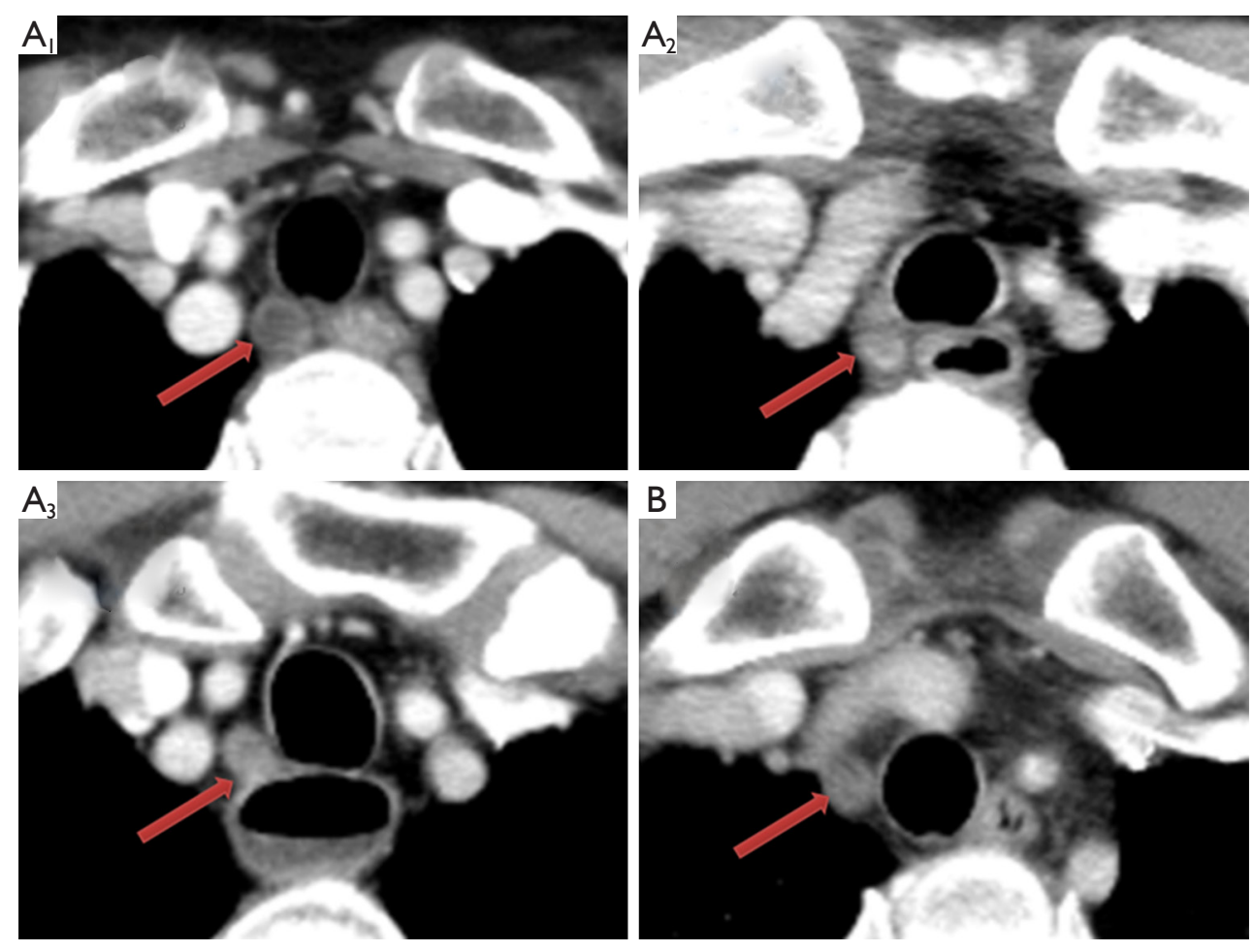

Figure 2 Distribution of metastatic right recurrent laryngeal nerve nodes on contrast-enhanced MSCT. A1, cervical right recurrent laryngeal nerve nodes in the tracheoesophageal groove; A2, junctional right recurrent laryngeal nerve nodes in the tracheoesophageal groove; A3, thoracic right recurrent laryngeal nerve nodes in the tracheoesophageal groove; B, right recurrent laryngeal nerve nodes located on the right side of the trachea away from the esophagus. MSCT, multi-slice computed tomography. 
Table 1 Characteristics of patients with and without Right recurrent laryngeal nerve nodes metastasis

\begin{tabular}{|c|c|c|c|}
\hline Characteristic & No. of patients (RRLNN-), n (\%) & No. of patients (RRLNN+), n (\%) & $P$ value \\
\hline Male & $363(5.7)$ & $99(15.8)$ & \\
\hline Female & $137(21.8)$ & $29(4.6)$ & \\
\hline Tumor location & & & 0.003 \\
\hline Middle & $243(38.9)$ & $55(8.8)$ & \\
\hline Lower & $141(22.5)$ & $25(4.0)$ & \\
\hline Tumor depth & & & 0.001 \\
\hline pTis & $20(3.2)$ & $0(0.0)$ & \\
\hline рT2 & $126(20.1)$ & $33(5.3)$ & \\
\hline pT3-4a & $223(35.5)$ & 77 (12.3) & \\
\hline Histologic grade $^{\dagger}$ & & & 0.727 \\
\hline G1 & $49(7.8)$ & $12(1.9)$ & \\
\hline G2 & $198(31.5)$ & $56(8.9)$ & \\
\hline G3 & $183(29.1)$ & $58(9.2)$ & \\
\hline
\end{tabular}

${ }^{\dagger}, 72$ patients with GX were excluded. RRLNN-, right recurrent laryngeal nerve node negative; RRLNN+, right recurrent laryngeal nerve node positive.

differences among the categorical data. Receiver-operating characteristic (ROC) curve was constructed to calculate the area under the curve (AUC), specificity and sensitivity. The diameter of the short axis of the largest right recurrent laryngeal nerve node (DC201) was used as the test variable, and the pathological result of right recurrent laryngeal nerve node was used as the state variable. Youden index (sensitivity + specificity -1 ) was used to determine the optimal cut-off point. A two-sided $\mathrm{P}$ value $<0.05$ was considered to be statistically significant.

\section{Results}

\section{The lymph node ratio of station C201 was higher than that of other stations}

Patient characteristics are shown in Table 1 . The median age was 60 years (range, $38-85$ years). The proportions of stage $\mathrm{pN} 0, \mathrm{pN} 1, \mathrm{pN} 2$ and $\mathrm{pN} 3$ patients were $56.8 \%, 26.3 \%$, $13.7 \%$ and $3.2 \%$, respectively. A total of 19062 lymph nodes were dissected, with an average of $30.4 \pm 14.4$ lymph nodes per case, including 1878 right recurrent laryngeal nerve nodes, with an average of $3.0 \pm 2.4$ lymph nodes. A total of 750 metastatic lymph nodes were dissected, including 199 metastatic right recurrent laryngeal nerve nodes. The lymph node ratio is defined as the total number of dissected nodes divided by the number of metastatic nodes. The lymph node ratio of right recurrent laryngeal nerve nodes, paracadial nodes, paraesophageal lymph nodes, left recurrent laryngeal nerve nodes, left gastric nodes, subcarinal nodes and diaphragmatic nodes were all greater than $1 \%$. The lymph node ratio was $10.6 \%, 5.0 \%, 4.9 \%$, $4.4 \%, 4.2 \%, 2.5 \%$ and $1.0 \%$, respectively. The lymph node ratio of station $\mathrm{C} 201$ was significantly higher than that of lymph nodes in other stations $(10.6 \%$ vs. $3.2 \%, \mathrm{P}<0.001)$.

\section{Tumor location and tumor depth are related to right recurrent laryngeal nerve node metastasis}

The correlation between gender, tumor location, tumor depth, histologic grade and lymph nodes was shown in 
Table 1. Right recurrent laryngeal nerve node metastasis rate of upper thoracic esophageal squamous cell carcinoma was higher than that of middle and lower thoracic esophageal squamous cell carcinoma $(\mathrm{P}=0.003)$. Right recurrent laryngeal nerve node metastasis was rare in patients with pT1a, but more in patients with pT1b, pT2, pT3 and pT4a. There was no statistically significant difference with regard to the right recurrent laryngeal nerve node metastasis rate between patients with pT1b, pT2 and pT3 $(\mathrm{P}=0.145)$, nor between patients with middle thoracic and lower thoracic esophageal squamous cell carcinoma $(\mathrm{P}=0.353)$.

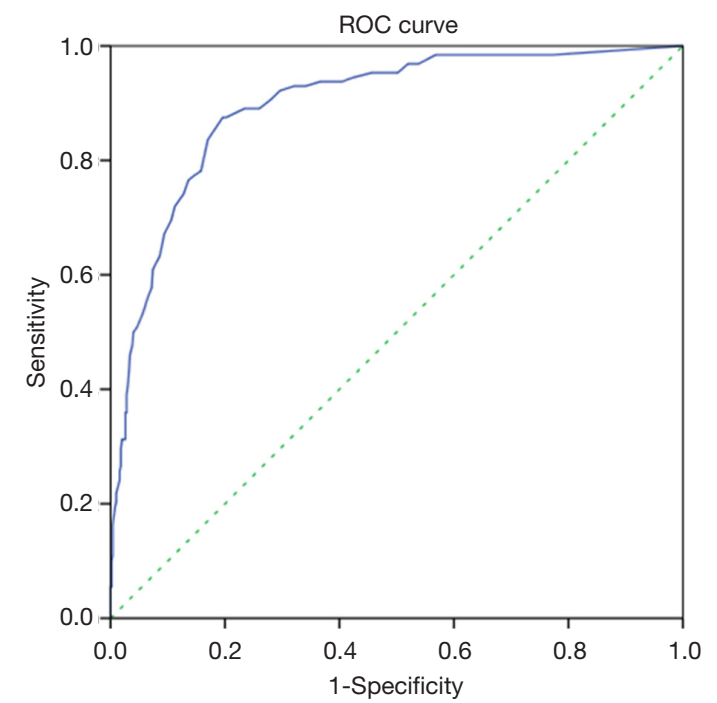

Figure 3 ROC curve for right recurrent laryngeal nerve nodes in thoracic esophageal squamous cell carcinoma. ROC, receiver operating characteristic.
This suggests that upper thoracic esophageal tumor and tumor breakthrough of submucosa are risk factors for right recurrent laryngeal nerve node metastasis in thoracic esophageal squamous cell carcinoma.

\section{The detection rate of right recurrent laryngeal nerve node metastasis by EUS was only $33.3 \%$}

Of all the 236 patients from SYSUCC who attempted EUS, 36 failed to complete EUS due to esophageal stenosis $(n=35)$ or suspected esophageal perforation $(n=1)$. Among those who successfully completed EUS, 54 had right recurrent laryngeal nerve node metastasis, among which only 18 were found to have lymph nodes within $23 \mathrm{~cm}$ from the incisors.

\section{The optimal cut-off point of DC201 was $5.4 \mathrm{~mm}$ instead of $10 \mathrm{~mm}$}

Of the 628 patients enrolled, 128 had right recurrent laryngeal nerve node metastases. ROC curve analysis revealed that the optimal cut-off point of DC201 was $5.4 \mathrm{~mm}$, with AUC of 0.896 (Figure 3), sensitivity of $87.5 \%$, specificity of $80.4 \%$, accuracy of $81.8 \%$, and the Youden index of 0.679. When the cut-off point of DC201 was set to $10.0 \mathrm{~mm}$, sensitivity, specificity, accuracy and the Youden index were $14.1 \%, 99.6 \%, 82.2 \%$, and 0.137 respectively. The diagnostic capabilities of DC201 at different cut-off points were summarized in Table 2. We observed that the probability of right recurrent laryngeal nerve node metastasis was 3.2\% (12/372) when DC201 $<5 \mathrm{~mm}$, but it increased to $45.3 \%(116 / 256)$ when DC201 $\geq 5 \mathrm{~mm}$.

Table 2 Diagnostic efficacy of DC201 with different cut-off points

\begin{tabular}{llllc}
\hline Cut-off point, $\mathrm{mm}$ & Sensitivity (\%) & Specificity (\%) & Accuracy (\%) & YI \\
\hline 4 & $96.9(124 / 128)$ & $48.0(240 / 500)$ & $58.0(364 / 628)$ & 0.449 \\
5 & $90.6(116 / 128)$ & $72.0(360 / 500)$ & $74.4(476 / 628)$ & 0.626 \\
5.4 & $87.5(112 / 128)$ & $80.4(402 / 500)$ & $81.8(514 / 628)$ & 0.679 \\
6 & $71.9(92 / 128)$ & $88.8(444 / 500)$ & $85.4(536 / 628)$ & 0.607 \\
7 & $50.0(64 / 128)$ & $96.0(480 / 500)$ & $86.6(544 / 628)$ & 0.460 \\
8 & $31.3(40 / 128)$ & $97.4(487 / 500)$ & $83.9(527 / 628)$ & 0.287 \\
9 & $21.1(27 / 128)$ & $99.0(495 / 500)$ & $83.1(522 / 628)$ & 0.201 \\
10 & $14.1(18 / 128)$ & $99.6(498 / 500)$ & $82.2(516 / 628)$ & 0.137 \\
\hline
\end{tabular}

DC201, the diameter of the short axis of the largest right recurrent laryngeal nerve node; YI, Youden Index. 


\section{The largest right recurrent laryngeal nerve nodes were mainly located in the tracheoesophageal groove and/or above the suprasternal notch}

The anatomical distribution of the largest right recurrent laryngeal nerve nodes in 128 patients with right recurrent laryngeal nerve node metastasis was also analyzed. Two patients were further excluded since their right recurrent laryngeal nerve nodes were too small to be clearly visualized even on contrast-enhanced MSCT. The number of cervical, junctional and thoracic maximal right recurrent laryngeal nerve nodes was 71, 20 and 35, respectively. On the transverse plane, the largest right recurrent laryngeal nerve nodes were located within $\angle \alpha$ in 124 patients (Figure 1), 108 of which were located in the tracheoesophageal groove (Figure 2).

\section{Discussion}

Esophageal lymphatic vessels run longitudinally in the lamina propria and the submucosa and circumferentially in the intermuscular space $(16,17)$. The longitudinal submucosal lymphatic network run along the right tracheoesophageal groove and is continuous with the lymphatics of the pharynx and the stomach (18). Only the right recurrent laryngeal nerve nodes have been previously extensively investigated and confirmed to be connected directly to the submucosal lymphatic plexus $(16,18)$. This anatomic structure underlies why right recurrent laryngeal nerve nodes have a higher rate of metastasis in thoracic esophageal squamous cell carcinoma. In line with this, our finding corroborated that the lymph node ratio of station C201 was significantly higher than that of lymph nodes in other stations.

The accuracy of clinical diagnosis of lymph nodes in esophageal cancer remains suboptimal $(2,19,20)$. Chest and abdominal CT scan with intravenous contrast and fluorine-18-fluoro-2-deoxy-D-glucose positron emission tomography (PET)/CT imaging are commonly employed imaging modalities to evaluate lymph nodes status. Unfortunately, CT and PET/CT imaging are curtailed by their low accuracy for detecting lymph node metastasis (21-27). Although recent developments in magnetic resonance (MR) imaging techniques have resulted in improved imaging quality and diagnostic accuracy of lymph node metastasis (28-30), this modality is seldomly used for the staging of patients with esophageal cancer. EUS has been shown to be superior to CT in detecting lymph node metastases, but the sensitivity and specificity vary dramatically depending on anatomic location $(22,31,32)$. In this study, the detection rate of right recurrent laryngeal nerve node metastasis by EUS was only $33.3 \%$, which is congruent with a prior study by Shan et al. that reported a sensitivity of merely $32.4 \%$ of EUS for the evaluation of right recurrent laryngeal nerve node metastasis (33). There are mainly 3 reasons accounting for this poor sensitivity. First, right recurrent laryngeal nerve nodes are obscured by the trachea in a subset of cases (more than $12.5 \%$ in our study). Second, right recurrent laryngeal nerve nodes are relatively smaller than those in other stations, so they are prone to be missed by EUS. Finally, the accuracy of EUS was also influenced by operator experience. Among the 54 patients who underwent EUS examination and had right recurrent laryngeal nerve nodes metastasis, 46 patients had DC201 $\geq 5.4 \mathrm{~mm}$, accounting for $85.2 \%$. It is obvious that under the new standard, MSCT is better than EUS in detecting right recurrent laryngeal nerve nodes. Due to the high cost of PET/CT examination and its mediocre performance in clinical $\mathrm{N}$ staging, it is rarely used in the evaluation of operable patients, so we do not have reliable data on PET/CT in this group of patients.

In clinical practice, regional lymph nodes are only suspicious for tumor involvement when being round in shape and/or $>10 \mathrm{~mm}$ in short axis diameter. This may underscore the notoriously poor accuracy of CT in diagnosing lymph node metastasis. As illustrated in the present study, the sensitivity of CT in diagnosing right recurrent laryngeal nerve node metastasis was only $14.1 \%$ when short axis diameter $\geq 10 \mathrm{~mm}$ was adopted as the criteria for tumor involvement. However, ROC curve indicated a good correlation between right recurrent laryngeal nerve node metastasis and DC201. When DC201 $\geq 5.4 \mathrm{~mm}$ was applied to define metastasis, the sensitivity, specificity and accuracy improved dramatically. We also analyzed data from SYSUCC and ACHZZU separately, and their AUC, optimal cut-off point, sensitivity and specificity was 0.851 , $5.2 \mathrm{~mm}, 87.5 \%, 72.1 \%$ and $0.927,5.5 \mathrm{~mm}, 87.5 \%, 80.4 \%$ respectively. This minor difference could potentially be explained by the fact that a larger percentage of patients at ACHZZU were excluded because of neoadjuvant therapy. The sensitivity and specificity of different cut-off points are listed in Table 2. We found that the sensitivity was $96.9 \%$ when the cut-off point was $4 \mathrm{~mm}$, and the specificity was $96.0 \%$ when the cut-off point was $7 \mathrm{~mm}$. This suggests that in clinical practice, if we find DC201 $<4 \mathrm{~mm}$, we can consider it as negative right recurrent laryngeal nerve node; 
if we find DC201 $\geq 7 \mathrm{~mm}$, we can consider it as positive right recurrent laryngeal nerve node.

Fine-needle aspiration [FNA] could aid in improving the sensitivity and specificity of EUS in evaluating $\mathrm{N}$ stage disease (34). Lymph nodes with suspected metastasis can be identified by endobronchial ultrasound-guided transbronchial needle aspiration (EBUS-TBNA) $(33,35)$. However, under what conditions lymph node biopsies are the best approach to be recommended? We observed that the probability of right recurrent laryngeal nerve node metastasis was minimal (3.2\%) when DC201 $<5 \mathrm{~mm}$, whereas it increased to $45.3 \%$ when DC201 $\geq 5 \mathrm{~mm}$. Therefore, we suggest that EUS-FNA or EBUSTBNA examination should be used to further clarify the status of right recurrent laryngeal nerve node when DC201 $\geq 5 \mathrm{~mm}$.

Station C201 in Chinese system and Station 2R in AJCC/UICC system are similar but different. We found that almost all the right recurrent laryngeal nerve nodes were distributed in $\angle \alpha$ (Figure 1), which suggested that Station C201 could better reflect the distribution of lymph nodes in this region than Station 2R. It also suggests that there is no need to dissect the anterior right side of the trachea to reduce the risk of right recurrent laryngeal nerve injury and right subclavian artery bleeding. Right recurrent laryngeal nerve nodes located in the tracheoesophageal groove were represent by A, Right recurrent laryngeal nerve nodes located on the right side of the trachea away from the esophagus were represent by B. Figures 1,2 clearly show that due to tracheal obstruction, B cannot be detected during EUS examination, which is the limitation of EUS. By analyzing the proportions of A1, A2 and A3, we found that right recurrent laryngeal nerve nodes were mainly located above the suprasternal notch, which suggested that we should not omit the right recurrent laryngeal nerve nodes above the suprasternal notch due to the difficulty in the operation.

The findings of the present study must be interpreted in acknowledging its limitations. First, this was a retrospective study, since all the removed right recurrent laryngeal nerve nodes were placed in a separate container for pathological examination, we knew how many right recurrent laryngeal nerve nodes were dissected and how many were metastatic in each patient, but we did not know which lymph nodes were metastatic, so we used DC201 as a predictor of right recurrent laryngeal nerve nodes status. Second, due to the lack of PET/CT data, the diagnostic efficacy of PET/ $\mathrm{CT}$ in the right recurrent laryngeal nerve lymph nodes of thoracic esophageal squamous cell carcinoma cannot be determined in this study. In a recent meta-analysis, the sensitivity of PET/CT in diagnosing esophageal squamous cell carcinoma lymph nodes was only $66 \%$, because small regional metastatic lymph nodes (range, $2-8 \mathrm{~mm}$ ) could not be detected by FDG-PET in cases of esophageal carcinoma (21-27). In view of the small size of right recurrent laryngeal nerve nodes in esophageal squamous cell carcinoma, we predicted that $\mathrm{PET/CT}$ would not have a significant advantage over CT in the diagnosis of right recurrent laryngeal nerve nodes. Last, there is the potential for a significant bias in this study because many patients who had obvious locoregional metastases underwent neoadjuvant treatment and were excluded from this study. Therefore, we suggest that DC201 $\geq 6 \mathrm{~mm}$ should replace DC201 $\geq 5.4 \mathrm{~mm}$ as the diagnostic criterion for positive right recurrent laryngeal nerve nodes, so as to make up for the bias of this study.

In conclusion, we found that the diagnostic efficacy of contrast-enhanced MSCT to evaluate right recurrent laryngeal nerve node status with a criterion of DC201 $\geq 6.0 \mathrm{~mm}$ was significantly higher than that when DC201 $\geq 10 \mathrm{~mm}$ was set as the criteria. Additional prospective studies are needed to conclusively confirm our results.

\section{Acknowledgments}

We thank all colleagues at SYSUCC and ACHZU who were involved in performing the treatments described in the current study.

Funding: None.

\section{Footnote}

Reporting Checklist: The authors have completed the STROBE reporting checklist. Available at http://dx.doi. org/10.21037/jtd-20-774

Data Sharing Statement: Available at http://dx.doi. org/10.21037/jtd-20-774

Peer Review File: Available at http://dx.doi.org/10.21037/jtd20-774

Conflicts of Interest: All authors have completed the ICMJE uniform disclosure form (available at http://dx.doi. org/10.21037/jtd-20-774). The authors have no conflicts of interest to declare. 
Ethical statement: The authors are accountable for all aspects of the work in ensuring that questions related to the accuracy or integrity of any part of the work are appropriately investigated and resolved. The trial was conducted in accordance with the Declaration of Helsinki (as revised in 2013) and the Harmonized Tripartite Guideline for Good Clinical Practice from the International Conference on Harmonization. This study was reviewed and approved by the Sun Yat-sen University Cancer Center and the Affiliated Cancer Hospital of Zhengzhou University. The approval number is waived because of the retrospective nature of the study. Informed consents were waived due to its retrospective nature.

Open Access Statement: This is an Open Access article distributed in accordance with the Creative Commons Attribution-NonCommercial-NoDerivs 4.0 International License (CC BY-NC-ND 4.0), which permits the noncommercial replication and distribution of the article with the strict proviso that no changes or edits are made and the original work is properly cited (including links to both the formal publication through the relevant DOI and the license). See: https://creativecommons.org/licenses/by-nc-nd/4.0/.

\section{References}

1. Rice TW, Apperson-Hansen C, DiPaola LM, et al. Worldwide Esophageal Cancer Collaboration: clinical staging data. Dis Esophagus 2016;29:707-14.

2. Rice TW, Ishwaran $\mathrm{H}$, Blackstone $\mathrm{EH}$, et al. Recommendations for clinical staging (cTNM) of cancer of the esophagus and esophagogastric junction for the 8th edition AJCC/UICC staging manuals. Dis Esophagus 2016;29:913-9.

3. Rice TW, Ishwaran H, Hofstetter WL, et al. Recommendations for pathologic staging (pTNM) of cancer of the esophagus and esophagogastric junction for the 8th edition AJCC/UICC staging manuals. Dis Esophagus 2016;29:897-905.

4. Glazer GM, Gross BH, Quint LE, et al. Normal mediastinal lymph nodes: number and size according to American Thoracic Society mapping. AJR Am J Roentgenol 1985;144:261-5.

5. Kiyono K, Sone S, Sakai F, et al. The number and size of normal mediastinal lymph nodes: a postmortem study. AJR Am J Roentgenol 1988;150:771-6.

6. Schmidt AF, Jr., Rodrigues OR, Matheus RS, et al. Mediastinal lymph node distribution, size and number: definitions based on an anatomical study. J Bras Pneumol 2007;33:134-40.

7. Li H, Fang W, Yu Z, et al. Chinese expert consensus on mediastinal lymph node dissection in esophagectomy for esophageal cancer (2017 edition). J Thorac Dis 2018;10:2481-9.

8. Rice TW, Ishwaran H, Ferguson MK, et al. Cancer of the Esophagus and Esophagogastric Junction: An Eighth Edition Staging Primer. J Thorac Oncol 2017;12:36-42.

9. Soeno T, Harada H, Hosoda K, et al. Lymph Node Progression and Optimized Node Dissection of Middle Thoracic Esophageal Squamous Cell Carcinoma in the Latest Therapeutic Surgical Strategy. Ann Surg Oncol 2019;26:996-1004.

10. Akiyama H, Tsurumaru M, Udagawa $\mathrm{H}$, et al. Radical lymph node dissection for cancer of the thoracic esophagus. Ann Surg 1994;220:364-72; discussion 372-3.

11. Tsurumaru M, Kajiyama Y, Udagawa H, et al. Outcomes of extended lymph node dissection for squamous cell carcinoma of the thoracic esophagus. Ann Thorac Cardiovasc Surg 2001;7:325-9.

12. Koyanagi K, Igaki H, Iwabu J, et al. Recurrent Laryngeal Nerve Paralysis after Esophagectomy: Respiratory Complications and Role of Nerve Reconstruction. Tohoku J Exp Med 2015;237:1-8.

13. Hiranyatheb P, Osugi H. Radical lymphadenectomy in esophageal cancer: from the past to the present. Dis Esophagus 2015;28:68-77.

14. Wright CD, Zeitels SM. Recurrent laryngeal nerve injuries after esophagectomy. Thorac Surg Clin 2006;16: 23-33, v.

15. Matsuda S, Takeuchi H, Kawakubo H, et al. Three-field lymph node dissection in esophageal cancer surgery. J Thorac Dis 2017;9:S731-40.

16. Wang Y, Zhu L, Xia W, et al. Anatomy of lymphatic drainage of the esophagus and lymph node metastasis of thoracic esophageal cancer. Cancer Manag Res 2018;10:6295-303.

17. Tachimori Y. Pattern of lymph node metastases of squamous cell esophageal cancer based on the anatomical lymphatic drainage system: efficacy of lymph node dissection according to tumor location. J Thorac Dis 2017;9:S724-30.

18. Mizutani M, Murakami G, Nawata S, et al. Anatomy of right recurrent nerve node: why does early metastasis of esophageal cancer occur in it?. Surg Radiol Anat 2006;28:333-8.

19. Gaur P, Sepesi B, Hofstetter WL, et al. A clinical nomogram predicting pathologic lymph node involvement 
in esophageal cancer patients. Ann Surg 2010;252:611-7.

20. Yokota T, Igaki H, Kato K, et al. Accuracy of preoperative diagnosis of lymph node metastasis for thoracic esophageal cancer patients from JCOG9907 trial. Int J Clin Oncol 2016;21:283-8.

21. van Vliet EP, Heijenbrok-Kal MH, Hunink MG, et al. Staging investigations for oesophageal cancer: a metaanalysis. Br J Cancer 2008;98:547-57.

22. Kim TJ, Kim HY, Lee KW, et al. Multimodality assessment of esophageal cancer: preoperative staging and monitoring of response to therapy. Radiographics 2009;29:403-21.

23. Choi J, Kim SG, Kim JS, et al. Comparison of endoscopic ultrasonography (EUS), positron emission tomography (PET), and computed tomography (CT) in the preoperative locoregional staging of resectable esophageal cancer. Surg Endosc 2010;24:1380-6.

24. Walker AJ, Spier BJ, Perlman SB, et al. Integrated PET/ CT fusion imaging and endoscopic ultrasound in the preoperative staging and evaluation of esophageal cancer. Mol Imaging Biol 2011;13:166-71.

25. Kim JJ, Park JK, Moon SW. Usefulness of positron emission tomography-computed tomography in preoperative evaluation of intra-thoracic esophageal cancer. Thorac Cancer 2015;6:687-94.

26. Jiang C, Chen Y, Zhu Y, et al. Systematic review and meta-analysis of the accuracy of 18F-FDG PET/CT for detection of regional lymph node metastasis in esophageal squamous cell carcinoma. J Thorac Dis 2018;10:6066-76.

27. Hu J, Zhu D, Yang Y. Diagnostic value of $18 \mathrm{~F}$-fluorodeoxyglucose positron-emission tomography/ computed tomography for preoperative lymph node metastasis of esophageal cancer: A meta-analysis. Medicine
(Baltimore) 2018;97:e13722 .

28. Yamada I, Hikishima K, Miyasaka N, et al. Esophageal carcinoma: Evaluation with q-space diffusion-weighted MR imaging ex vivo. Magn Reson Med 2015;73:2262-73.

29. Yamada I, Miyasaka N, Hikishima K, et al. Ultra-highresolution MR imaging of esophageal carcinoma at ultra-high field strength (7.0T) ex vivo: correlation with histopathologic findings. Magn Reson Imaging 2015;33:413-9.

30. Qu J, Shen C, Qin J, et al. The MR radiomic signature can predict preoperative lymph node metastasis in patients with esophageal cancer. Eur Radiol 2019;29:906-914.

31. Takizawa K, Matsuda T, Kozu T, et al. Lymph node staging in esophageal squamous cell carcinoma: a comparative study of endoscopic ultrasonography versus computed tomography. J Gastroenterol Hepatol 2009;24:1687-91.

32. Luo LN, He LJ, Gao XY, et al. Endoscopic Ultrasound for Preoperative Esophageal Squamous Cell Carcinoma: a Meta-Analysis. PLoS One 2016;11:e0158373.

33. Shan HB, Zhang R, Li Y, et al. Application of Endobronchial Ultrasonography for the Preoperative Detecting Recurrent Laryngeal Nerve Lymph Node Metastasis of Esophageal Cancer. PloS one 2015;10:e0137400.

34. Puli SR, Reddy JB, Bechtold ML, et al. Staging accuracy of esophageal cancer by endoscopic ultrasound: a metaanalysis and systematic review. World J Gastroenterol 2008;14:1479-90.

35. Liberman M, Hanna N, Duranceau A, et al. Endobronchial ultrasonography added to endoscopic ultrasonography improves staging in esophageal cancer. The Annals of thoracic surgery 2013;96:232-6: discussion 236-8.
Cite this article as: $\mathrm{Li} Z \mathrm{ZX}, \mathrm{Li} \mathrm{XD}$, Liu XB, Xing WQ, Sun HB, Wang ZF, Zhang RX, Li Y. Clinical evaluation of right recurrent laryngeal nerve nodes in thoracic esophageal squamous cell carcinoma. J Thorac Dis 2020;12(7):3622-3630. doi: 10.21037/jtd-20-774 